\title{
Texture Roughness Estimation Using Dynamic Tactile Sensing
}

\author{
Samuel Rispal, Axay K. Rana, and Vincent Duchaine \\ Dept. of Automated Manufacturing Engineering \\ École de Technologie Supérieure (ÉTS) \\ Montréal, Québec, Canada \\ e-mail: vincent.duchaine@etsmtl.ca
}

\begin{abstract}
Roughness estimation can help with improving tactile prehension and distinguishing slippage events during object manipulation with a robotic hand. Humans are able to estimate roughness from a small contact area with an object, and adapt manipulation strategies using this information [1]. In order to do the same with a robotic hand fitted with tactile sensors, this article focuses on how to estimate roughness with data from a tactile sensor. We propose a learning algorithm that estimates roughness on a scale from 1 to 5 , which was inspired by human tactile capabilities. For more adapted parameters values, this algorithm is optimized with a genetic algorithm. To initialize the scale, we asked 30 people to classify 25 textures on a roughness scale from 1 to 5 . The results were used to feed the learning algorithm. After testing our algorithm on those 25 textures, we conclude that even if there are small errors on certain textures, our algorithm is able to adapt itself to new textures and provide a roughness estimation that approximates the human one.
\end{abstract}

Keywords- Grasping; Tactile intelligence; Tactile sensing; texture recognition

\section{INTRODUCTION}

Humans' incredible ability to interact with unstructured environments relies greatly on their sensing capability. Indeed, the human hand possesses an amazingly large number of mechanoreceptors of several different types, which make it the most sensitive part of the human body [2]. This high concentration of sensory elements, along with their diversity, allows humans to construct a rich representation of the environment's properties (e.g. contact location, texture, object stiffness). This information can then be used to achieve excellent performance during manipulation. In order to approach or at least mimic this performance, robots used in assembly tasks will have to be equipped with a tactile sensory system that provides similar information [3].

Following this rationale, much progress has been done recently on giving the sense of touch to robots by designing novel tactile sensors. Most of these approaches typically use piezoresistivity [4], or capacitance [5], [6], [7] as a sensing principle, which provides only an image of the applied stress. Human hands have a complex combination of mechanoreceptors that are not only sensitive to pressure, but also to dynamic events such as the vibration that occurs at the fingertips when we touch, for example, a surface texture. In order to better match the human tactile capabilities, some recent studies have presented multimodal tactile

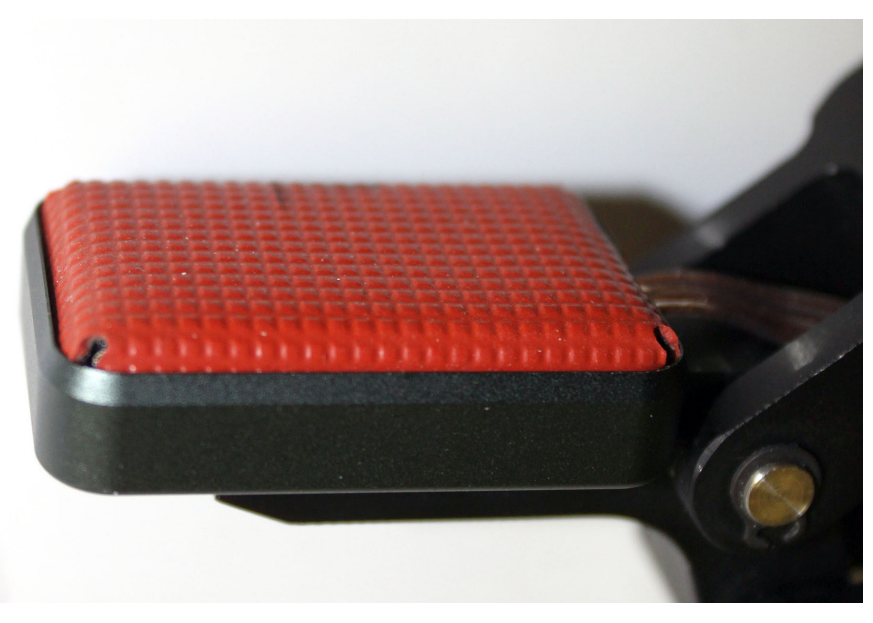

Fig. 1. Tactile sensor used for recognizing texture.

sensors that can be sensitive in both static and dynamic load conditions [8], [9], [10].

However, simply adding tactile sensors to robot hands alone is not sufficient to improve robots' grasping skills [11]. In order to have a real positive impact, these tactile sensors must be accompanied by algorithms that can translate their complex signals into high-level information, such as the recognition of object slippage [12], [13] or the estimation of grasp stability [14]. Another high-level cognitive skill that tactile sensing could provide robots with is the ability to recognize textures. Previous work done in our laboratory, as well as works carried out by other researchers [15], [16] and [17], have shown that it is possible for a robot to learn to recognizes textures by using tactile sensor data. Such a capability could be used as an object signature that could help the robot identify the object or estimate the object-hand friction properties in order to better control the grasping force without losing its grip on the object.

The objective of this work is directly linked to this goal. In this paper we propose a novel algorithm that uses a scale of roughness similar to the way a human being quantifies the perceived texture of an object upon contact. We intend to develop this scale as a way to encode these human-specific capabilities in order to use it on robots with touch sensing. We decided to use such an objective characterization as the 
roughness of a texture instead of a more strict technical definition, since there is still no consensus on how we can exactly characterize a texture [18]. Moreover, using some of the proposed definitions of the technical properties of a texture would have necessitated measuring the microgeometry of the textures (such as the depth and width of the striations, properties that are delicate to measure with actual tactile sensors). Human perception of textures, although being slightly relative from one person to an other, has been shown [19] to be a valuable and reliable source of information to characterize texture roughness properties.

The paper first presents the experimental setup used. Section 3 describes the experiments wherein 30 participants were ask to classify the perceived roughness of 25 different objects on a scale of 0 to 5 . Section 4 presents the classification algorithm used on the robots. Finally Section 5 present the classification results that were obtained by using the proposed algorithm on the same textures.

\section{EQUIPMENT USED IN THE EXPERIMENTS}

Two specific types of equipment were used for this study: 25 everyday life textures and a tactile sensor. We used the textures to compare the machine algorithm's output to human roughness perception. They were chosen to be a relatively exhaustive selection of everyday life textures, from the softest to the roughest. The sensor was used to make the acquisition on the textures for the learning algorithm.

\section{A. The 25 textures}

Our goal is to estimate the roughness of a texture with a learning algorithm that is comparable to human tactile perception. For our experiment, we used the 25 textures from everyday life presented in Fig. 2, and described as follows:

- (a) is a composite wood similar to the one used for tables or furniture.

- (b), (c), (d), (i), and (j) are plastics similar to the ones used in everyday life objects for better hand prehension (cell phones, printers, boxes...).

- (e), (f), (g) are sandpapers.

- (h) is a patterned leather used for cellphone cases.

- (k), (l), (m), (w) are standard papers.

- (n), (o), (p) are different smooth plastics.

- (q) is a metal sheet, like a brushed metal surface.

- (r) is like a mirror.

- (s) is wreath leather used for different design objects (pencil cases, bags...).

- (t) is a used file.

- (u) is wreath fabric that looks like synthetic leather objects.

- (v) is wreath plastic that looks like an anti-slip bathroom carpet.

- (y) is wreath metal that is used for microphones or loudspeakers.

All these textures will be tested by humans and the algorithm.

\section{B. Tactile sensor}

The sensor shown in Fig. 1 was used in our experiments. This sensor is a capacitive device able to acquire two types of data: normal stress and dynamic signals.

The first type consists of images of the normal stress applied on the sensor with a resolution of 28 taxels per phalange. The sampling frequency was set at about $59 \mathrm{~Hz}$ [9]. This capacitive sensor used a two layer microstructured silicone dielectric filled with nanoparticles of ferroelectric ceramic. This characteristic of the sensor enables a very broad measurement range while also making it highly sensitive to low forces $\left(10^{-4} \mathrm{~N}\right.$ per taxel).

The sensor also measures the stress rate applied on the sensor. Instead of differentiating the capacitance value over time, dynamic sensing is achieved here using a transimpedance amplifier that goes out of its equilibrium only in reaction of variation of the sensor capacitance. This make it very sensitive to any dynamic event, such as vibration, and allows us to have a sampling frequency considerably higher than for static measurements $(1000 \mathrm{~Hz}$ vs $25 \mathrm{~Hz})$. Characterizing the sensor's response to dynamic signals is more complex than for static load. We used a vibrotactile transducer called the Haptuator [20] and commercialized by Tactile Labs to validate the sensitivity of the sensor to vibration both in term of frequency and amplitude. During numerous tests, exceptional sensitivity was observed, exceeding human sensitivity to mechanical vibratory frequency and amplitude. Humans can distinguish frequencies in the $50-500 \mathrm{~Hz}$ range [21], which is the range of acquisition of our sensor. For amplitude, we carried out a simple test in which the Haptuator amplitude was set below the vibrational amplitude detectable by humans (performed on 10 subjects) at $250 \mathrm{~Hz}$. A peak of a clear amplitude on the FFT of the sensor signal was still clearly displayed.

\section{HUMAN ROUGHNESS QUANTIFICATION}

Different studies have tried to characterize roughness. Most of them define roughness using the microscopic variations on the surface [22], [23]. This approach is currently not possible with tactile sensor because of the sensitivity of the sensor. It can measure very small pressure differences on a surface but can't measure small geometrical variations. This is mainly due to the small geometrical scale and to the material used on the surface that is in contact with the objects. This is why we chose a different approach: trying to characterize roughness the way human do.

Human roughness perception is a complicated subject. Different human mechanoreceptors are involved in the process (SA I, PCs) [24] and the speed and strength of the way the finger slides on the texture influences its perception [25]. In order to obtain reliable results, we asked 30 participants to classify 25 textures with their own roughness perception. This section presents this experiment. In the next section, we will describe using these results to simulate the same experiment with a tactile sensor and a learning algorithm. 


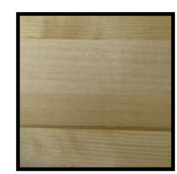

(a)

Composite

wood

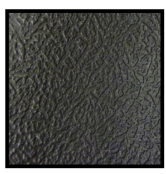

(j) Casted motifs plastic

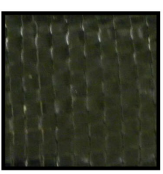

(s) Wreath leathe
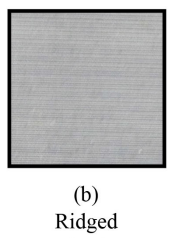

plastic

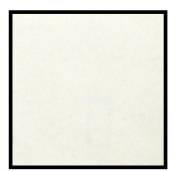

(k)

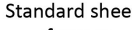

of paper

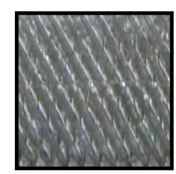

(t)
worn file

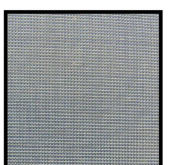

(c)

Protruding square motifs plastic

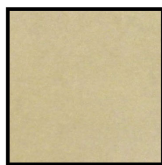

(I)

cardboard paper

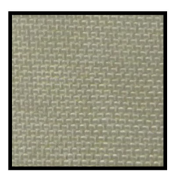

(u) Wreath fabric

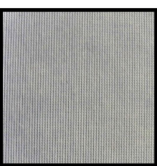

(d)

Sunken motifs plastic

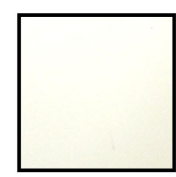

(m)

Photo paper

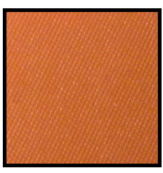

(v)

Sticky wreath

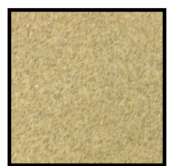

(e)

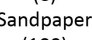

(100)

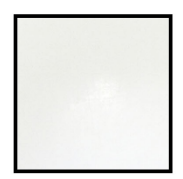

(n)

white smooth

plastic

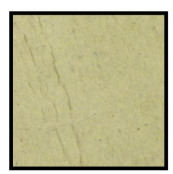

(w)

Worn

paper

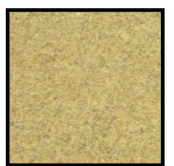

(f)

Sandpaper

(220)

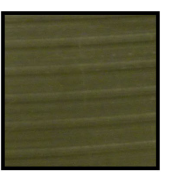

(o)

Scratched plastic

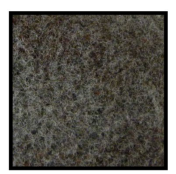

(x) Board brush

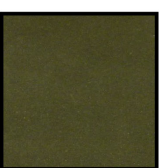

(g)

Sandpape

(400)

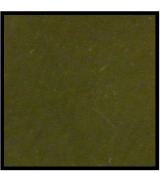

(p) Sticky plastic

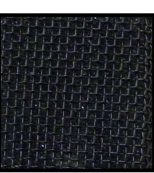

(y)

Wreath met

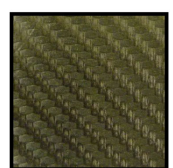

(h)

Leather with

motifs

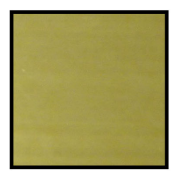

(q) Metal sheet

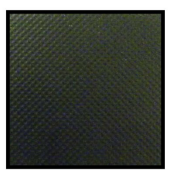

(i)

Protruding

circle motifs plastic

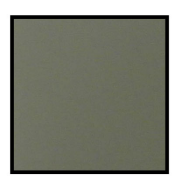

(r)

Silicium sheet

Fig. 2. Textures used for the experiments

\section{A. Experiment protocol}

The goal of this experiment is to classify 25 textures on a scale of 1 to 5 according to their roughness intensity. 30 people participated in the test. Half were men and half were women. 15 were 18-25 years old, 10 were 26-40 years old, and 5 were 41 years old and older. Half of these people were studying or working in sciences, with 7 of them in areas related to robotic tactile sensing.

When they entered the room, they sat at a table on which the 25 textures were placed. They each received a sheet with an empty chart for classifying the textures. After listening to a presentation on the different textures, they received some instructions:

- They can touch the textures, look at them, and handle the textures as needed.

- They should assign a roughness grade from 1 to 5 to each texture.

- The textures need to be fairly evenly distributed across the scale (4-6 textures per grade).

- They should be careful about noting the difference between roughness and stickiness.

- There is no time limit. They can take all the time they need.

\section{B. Results}

The participants need from 5 to 25 minutes to classify the 25 textures according to their roughness perception. Each participant used a different method to achieve his goal. Some classified the materials on 5 groups on the table, others gave a grade after one examination per texture and did a second checking tour. The last ones found the most and less rough textures and then completed the middle. When we asked people how they found the experiment, most of them were surprised by the complexity of this classification and didn't think they would give exactly the same results if they did the same experiment a second time.

The results of the experiment are shown in Fig. 3. For a better reading of the results, we chose to remove the grades that received less than 4 votes that have little impact. With the results, we observe an agreement on the extreme values (1 and 5). In the middle range, the participants differ frequently and sometimes the votes are on 3 or 4 different grades.

Fig. 4 presents another view of the results. The box represent the average plus/minus the standard deviation. We observe small boxes in the extremes and on some textures on the middle. But some boxes are on two grades. A comment given by many participants can explain that: "Sometimes, textures are not more or less rough, they are just different."

\section{Hypothesis for the simulation}

In the next section, we present a learning algorithm that estimates the roughness of textures using tactile sensing data. We use this section's results to test it. Some textures are used to optimize the algorithm and learn the roughness grades. The others are used to test the algorithm after the optimization to verify the efficiency of the algorithm.

We want to have 3 textures per grade to optimize the learning algorithm. We attempt to use the ones with the smaller standard deviation. The selected textures for optimizing and 


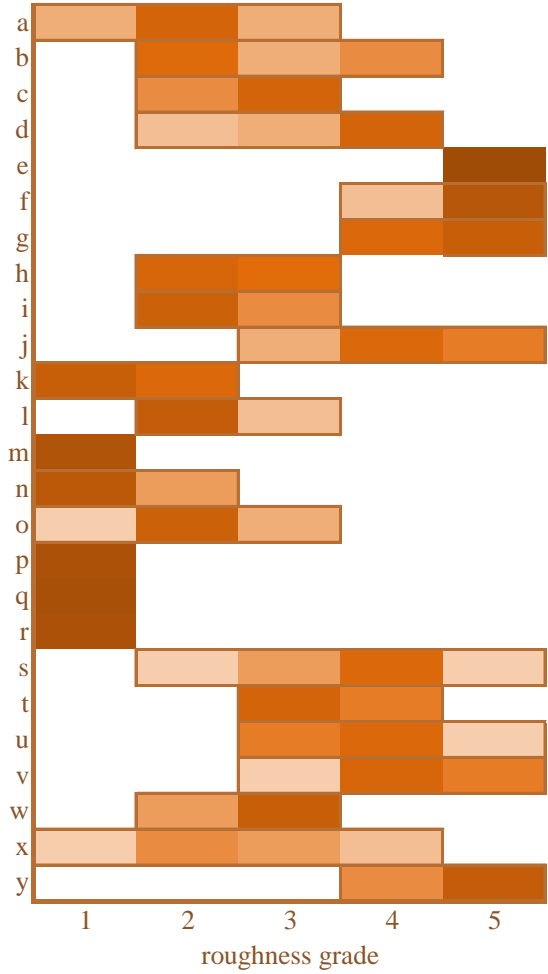

Number of votes per grade :

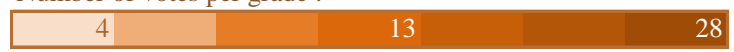

Fig. 3. Experiment results: number of participant votes per grade

testing the algorithm are detailed in Table I. We notice that textures like s) and $\mathrm{x}$ ) have quite large standard deviations, and in these cases it likely be particularly interesting to compare the participant's feedback to the results of the learning algorithm.

TABLE I

TEXTURES SELECTED TO FEED THE ALGORITHM

\begin{tabular}{|c|c|c|}
\hline $\begin{array}{c}\text { Roughness } \\
\text { grade }\end{array}$ & $\begin{array}{c}\text { Selected textures } \\
\text { for optimization }\end{array}$ & $\begin{array}{c}\text { Selected textures } \\
\text { for testing }\end{array}$ \\
\hline 1 & p), q), r) & k), m), n) \\
2 & a), l), o) & h), i), x) \\
3 & c), t), w) & b), d) \\
4 & j), u), v) & s) \\
5 & e), f), g) & y) \\
\hline
\end{tabular}

\section{ROUGHNESS QUANTIFICATION ALGORITHM}

As we previously saw, the sense of touch plays a very important role in the manipulation of objects, much more so than vision, which allows planning of movement [26]. It is particularly interesting that the manner in which a human seizes or catches an object depends on perception of characteristics such as fragility [27]. Knowing how to evaluate different characteristics of objects touched (texture, fragility, roughness, adhesiveness, etc.) is therefore very relevant to touch robotics. In this article, we focus on roughness

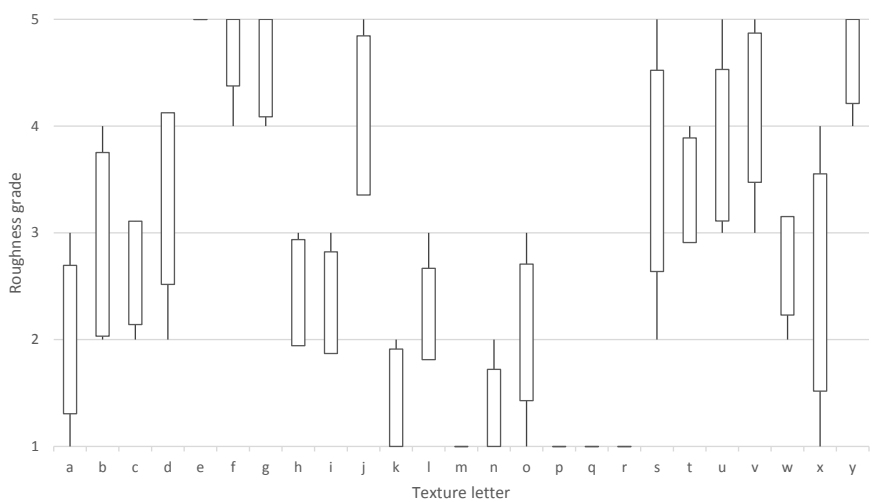

Fig. 4. Statistical view of the results with standard deviation box

estimation. Our goal is to propose an algorithm that could have a roughness estimation similar to human perception.

To create this algorithm, we were inspired by texture recognition algorithms.

When it comes to texture recognition algorithms, many studies have used capacitive sensors. Globally, texture discrimination over $90 \%$ correct has thus been achieved [28]. Two studies in particular attracted our attention: in the first of these, a finger-like probe containing multiple randomly placed sensors (static and dynamic) was used. After data pre-treatment, textures were distinguished using the main components of the Fourier transform in a learning algorithm. Eight textures were differentiated at three different speeds with $90 \%$ success using a majority vote [29] [30].In the second study, a statistical method called Bayesian inference was used, which functions using a relatively small set of training data. However, to confirm a texture, three movements were required. A BioTac probe with static and dynamic sensors (up to $2200 \mathrm{~Hz}$ ) was used. Over $95 \%$ of the 117 textures in the test were recognized [31] but the information acquisition step required a relatively long time to complete.

Various sensor technologies such as MEMS [32] [33] and opto-tactile sensors [34] have been tested in other studies.

The goal of our study is to propose an algorithm that estimates the roughness of each texture. For this, we have focused on three elements: $i$ the choice of movement, $i i$. data acquisition, pre-processing and extraction, and iii. learning algorithms. To optimize this algorithm, we used a genetic algorithm. The last part of this section details the genetic algorithm. Fig. 5 represents the steps of the algorithm.

\section{A. Exploratory motions}

The exploratory movement has a major impact on the quality of texture discrimination. Several studies have examined the implications of the choice of movement. Twodimensional linear motion has been chosen in many cases [30] [35]. Object typing is another approach that has been studied [36]. Finally, a thorough study of human texture recognition movements has been published [31]. The authors selected three movements in an attempt to discriminate 


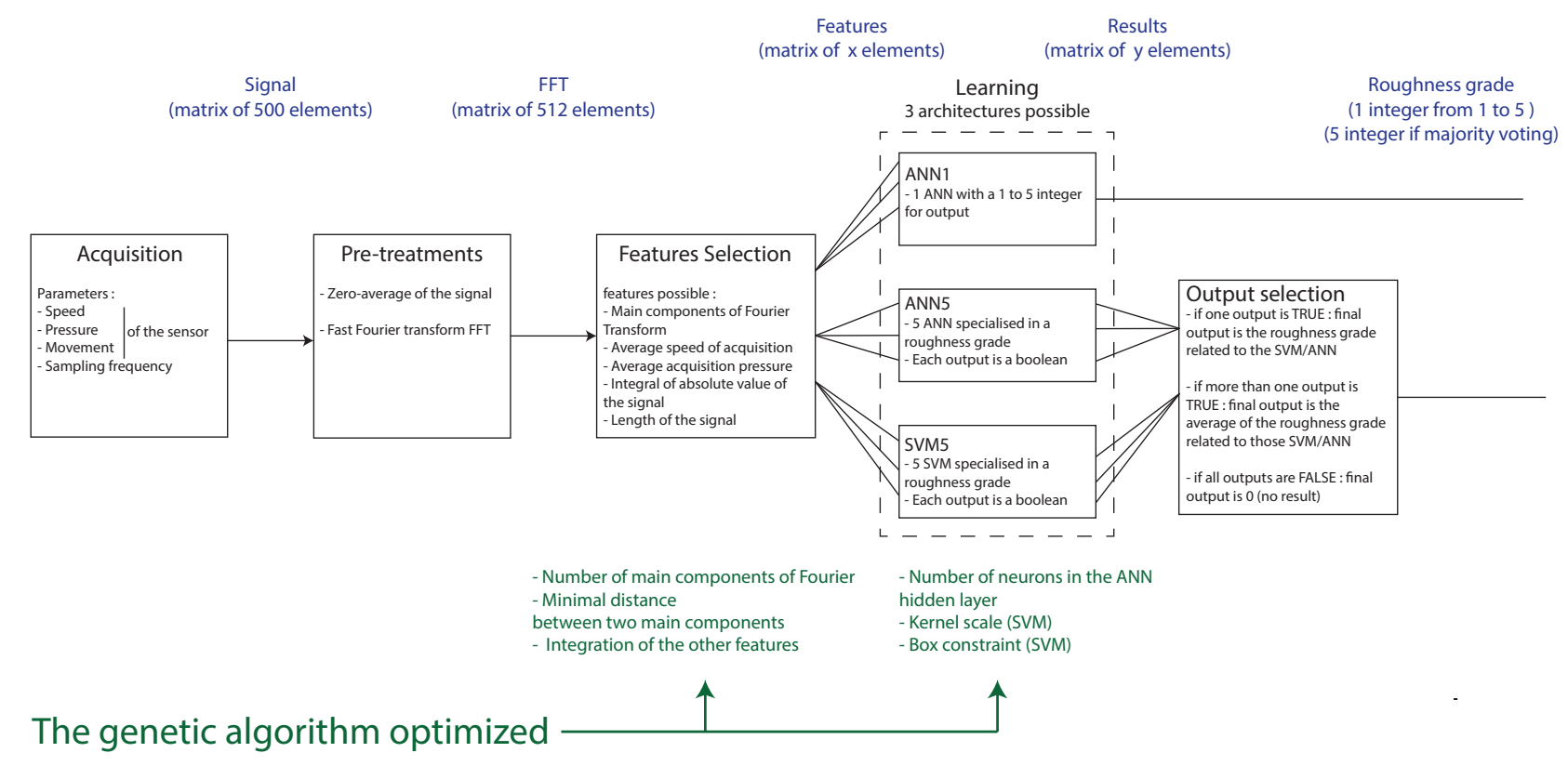

Fig. 5. Steps of the algorithm

textures using fewer learning data and very high-success-rate data.

For the purposes of our study, we selected one exploratory movement. Our exploratory movement is a short sliding linear movement in a single direction. This movement can be compared to the friction of the thumb on an object held in the hand. It's the movement we've seen the most during during the first experiment.

Human roughness perception is a complicated phenomenon. The speed and strength of the finger as it moves on the texture has an important impact on roughness perception [25][37]. Therefore we chose to make all our acquisitions at the same speed and strength.

\section{B. Features}

The choice of discriminants is particularly important since they determine the quality of roughness estimation. We need discriminants that represent the roughness of a texture without being specific to one texture. Before extracting discriminators, two pre-treatments are applied. The signal is first zero-averaged in order to obtain a Fourier transform with no null frequency component. The fast Fourier transform (FFT) that will extract the discriminants is then calculated in post-processing Matlab using the function fft. The duration of the input signal is from $500 \mathrm{~ms}$. The Fourier transform is calculated on 1024 values distributed equally between $0 \mathrm{~Hz}$ and $500 \mathrm{~Hz}$. Fig. 6 shows the signal and its Fourier transform.

When selecting the discriminants, most studies of capacitive sensors draw inspiration from sound processing using the main components of the Fourier transform. The main components are the maximum of Fourier Transform. A minimal distance between two main components is required. Several studies have also taken the speed and force applied by the robot into account. In our case, we study the impact of several discriminants and let the genetic algorithm determine whether to use them or to change their parameters. We describe the discriminants and their features below.

The main components of the Fourier transform: These components show the predominant frequencies in the material, which are directly related to the material surface properties (lines, grid, etc.) and are somewhat correlated with the speed of acquisition. Our algorithm detects the maximum in the Fourier transform. A tolerance margin imposes a minimal gap between the maxima. The user (or the genetic algorithm) selects the number of detected maxima and the code uses the frequency and amplitude of these peaks as the discriminant of the learning algorithm.

The average speed of acquisition: An estimate of the average speed of acquisition (scanning) is included in the learning algorithm in order to improve the anticipation of changes in the peak Fourier transform frequency.

The average acquisition pressure: The force applied to the sensor influences the amplitude of the various peaks of the Fourier transform.

The integral of the absolute value of the signal $i$ is calculated using equation 1 : 

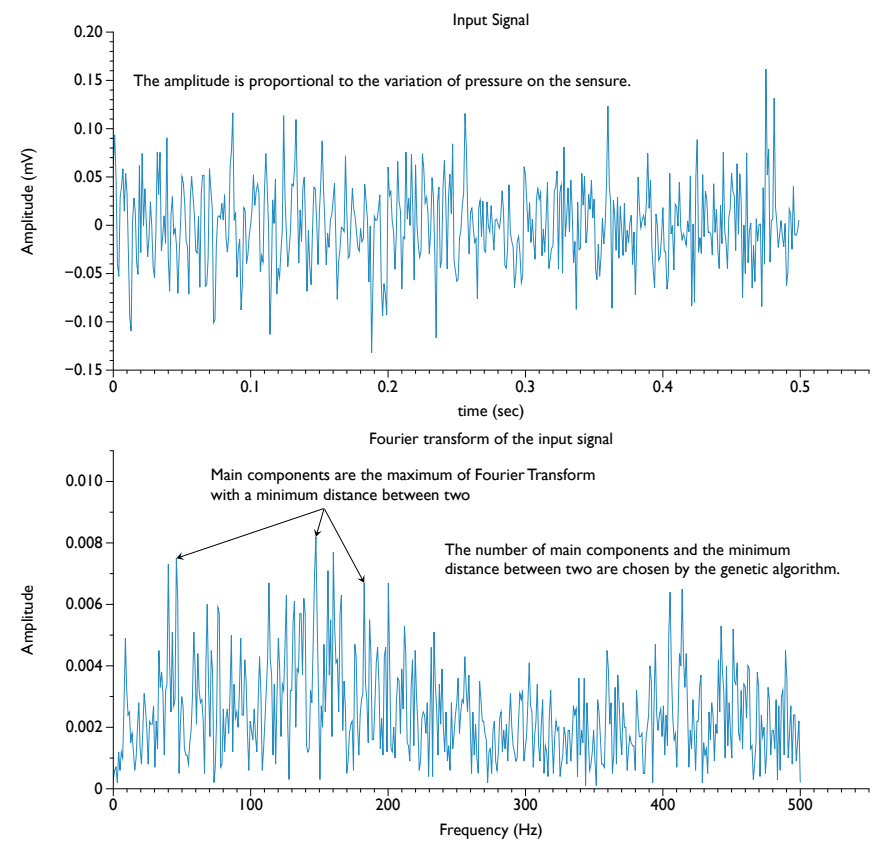

Fig. 6. Graphic of the input signal and its Fourier transform

$$
i=\int_{0}^{l}\left|s(t)^{2}\right| \mathrm{d} t
$$

In this formula, $l$ is the signal length and $s(t)$ represents the signal values. Combining the signal values in $s(t)$ enables better understanding of the signal and can be brought in line with the average force. It should allow differentiation of two signals that vary in intensity as well as low frequencies.

Signal length $l$ is calculated using equation 2 :

$$
l=\sum_{k=1}^{l} \sqrt{\left(t_{k+1}-t_{k}\right)^{2}+\left(s_{k+1}-s_{k}\right)^{2}}
$$

This index also provides information on signal amplitude and frequency.

\section{Neural Network}

The discriminants are then submitted to a learning algorithm. The learning algorithms selected to process the discriminants are among the most popular: artificial neural networks (ANN) and support vector machines (SVM). The number of inputs and the number of neurons in the hidden layer of the ANN depend on the genetic algorithm. The transfer function in the hidden layer is linear and the output neuron is a linear function. We propose two different ANN architectures: in the first one, called ANN 1, the output provides the roughness estimation with a number from 1 to 5 . In the second (ANN 5), we use the same structure the SVM (SVM 5). Instead of one, we train 5 ANN/SVM, each on specialized in one roughness grade (from 1 to 5). Each output is a boolean function. So the output of the learning algorithm is 5 boolean value. Three different actions are possible:

- one SVM/ANN output is TRUE: the result of the algorithm is the roughness grade related to this SVM.
- more than one SVM/ANN output is TRUE: the result of the algorithm is the average of the roughness grades related to those SVM/ANN.

- all the SVM/ANN output are FALSE: the result of the algorithm is 0 . It means the algorithm cannot find an answer for this input data.

\section{Optimizing with the genetic algorithm}

We now turn to the question of how to optimize the algorithm's variables such as the ANN or SVM configuration, the number of main FFT components, the choice of discriminators, etc., in order to obtain the best result. For this purpose, we used a standard genetic algorithm (GA) with 20 individuals and 30 generations.

To limit over-fitting, the algorithm separates the optimization database (cf III-C) into two equal parts: a learning portion, and a testing portion. The algorithm performs 20 different learning tasks, which are then tested against the test part of the optimization database. The fitness function returns the average of the results of learning and testing. At the end, we use the test database (cf III-C) to test our algorithm's efficiency.

The GA determines a set of variables for improving our learning algorithm:

1) Number of frequency peaks: The value of this variable ranges from 2 to 15 , and indicates the number of relevant peaks required to define the texture.

2) Minimum frequency between each peak: This value varies between 5 and $50 \mathrm{~Hz}$. The purpose of the spread is to filter noise produced by adjacent peaks, without affecting the major peaks.

3) Ranking by frequency or intensity: This variable determines whether the classification of peaks will be by intensity or by frequency prior to entry into the learning algorithm.

4) Signal integral: Used to determine whether or not the signal integral should be included as an input variable of the learning algorithm.

5) Signal length: Used to determine whether or not signal length should be included as an input variable of the learning algorithm.

The following three variables are with respect to the learning algorithm itself.

1) Number of neurons in the ANN hidden layer: This varies between 5 and 50 .

2) Box constraint (SVM): This ranges from $10^{-} 2$ to 10. This value limits the size of certain SVM vector coefficients and thereby establishes different borders.

3) Kernel scale (SVM): This ranges from $10^{-} 5$ to $10^{5}$. This variable changes the Kernel function scale, thus allowing non-linear transformations to be used.

\section{EXPERIMENTING THE LEARNING ALGORITHM}

In this section, we describe how we tested the algorithm presented in Section IV. We used the results of Section III to initialize the algorithm. 


\section{A. Methodology}

We tested our algorithm using the result of the experiment from the Section III. In this context, we created a database of tactile information using our tactile sensor. We rubbed the sensor on each texture presented in the Section II-A. The speed, the pressure and the direction is constant. The average speed is $0.15 \mathrm{~m} / \mathrm{s}$ and the average strength is 2.5 $\mathrm{N}$. When the texture have some ridges, the chosen direction is against the ridges. We acquired 200 samples per texture, representing a database of 5000 samples. The duration of each scan is $700 \mathrm{~ms}$.

Our database is divided in two categories: the optimization dataset and the test dataset. Each texture received a category in Section III-C, following the roughness estimation experience with humans. The optimization dataset is used to feed and test the learning algorithm during the genetic algorithm optimization. When all optimizations are done, we use the test dataset to test the results. This dataset samples come from textures unused during the optimization phase.

In this experiment, we want to compare the three different learning algorithm architecture to the roughness estimation experiment made by humans (Section III).

\section{B. Results}

The genetic algorithm execution lasts 6 hours per architecture. The algorithm sends back the optimized ANN or SVM and the roughness estimation for each sample. It means there is 5000 answers to process. For a brief but meaningful presentation of the results, we divide them in two parts: the results from samples used for optimization and the ones from samples used for testing. The first main piece of information is the average error between the the results and the expected value. This value estimates how close the algorithm perception is compared to the human one. The second main piece of information is the average standard deviation per texture. This value estimates the amplitude of the results, i.e., how far they can be from the average. Table II sums up the results of the experiment.

TABLE II

SUMMARY OF THE EXPERIMENT

\begin{tabular}{|c|l|c|c|c|}
\cline { 3 - 4 } \multicolumn{2}{c|}{} & ANN 1 & ANN 5 & SVM 5 \\
\hline Optimization & Average error & 0.65 & 0.24 & 0.25 \\
set & Standard deviation & 0.59 & 0.60 & 0.64 \\
\hline Testing & Average error & 1.13 & 1.02 & 1.3 \\
set & Standard deviation & 0.59 & 0.55 & 0.77 \\
\hline
\end{tabular}

Globally, Table II shows that a learning with good result is possible, but the testing average error isn't under 1 roughness grade. Between the three architectures, the ANN 5 architecture works a little bit better than the others. Indeed, ANN 1 architecture have some troubles with the learning part. The average error is higher than other architectures. In SVM 5, the problem is with the testing set. The error is higher than others, we observe some overfitting.

Figure 7 compares the results of the simulation to the results of the experience with human perception. In green,

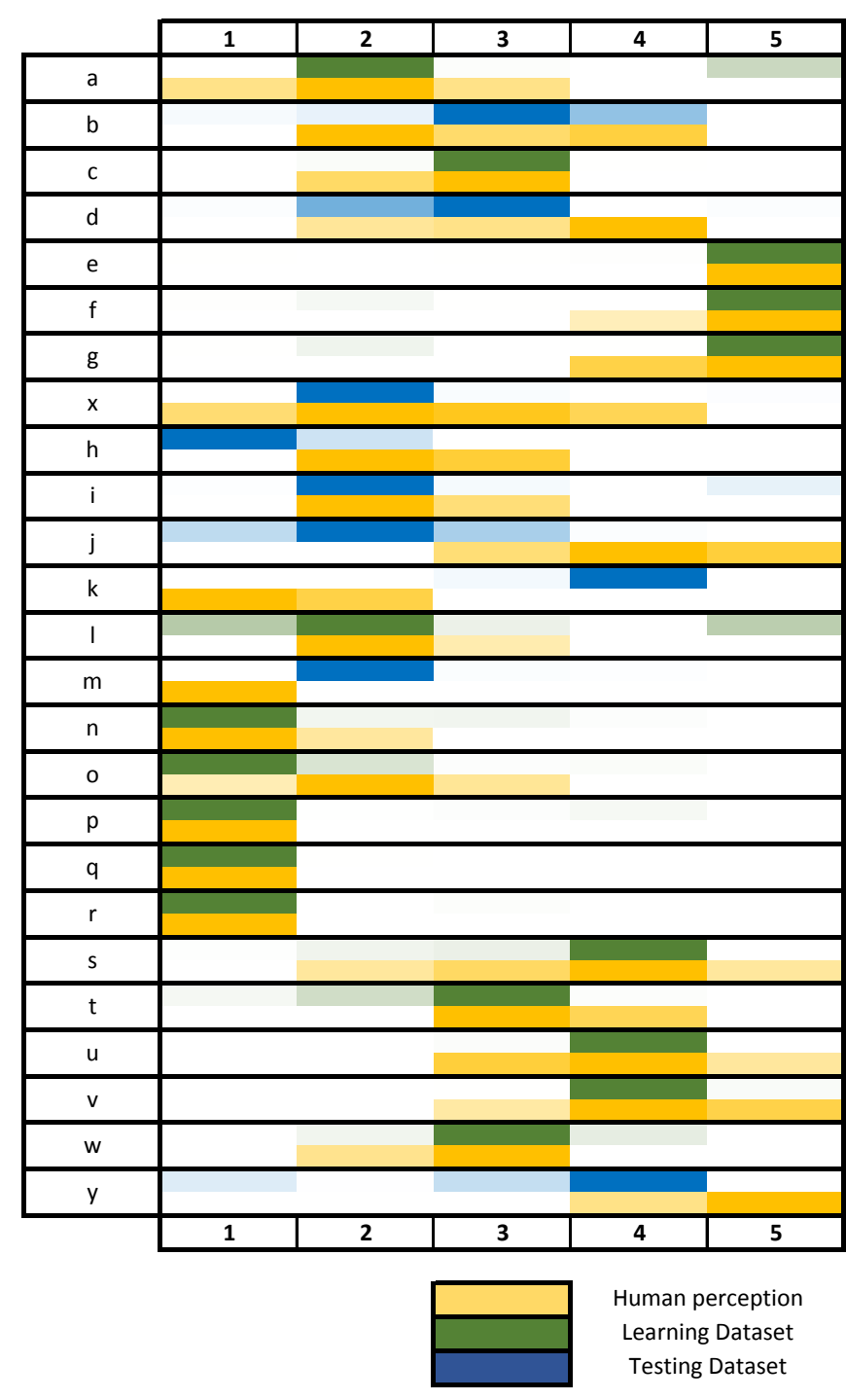

Fig. 7. Results with ANN 5 architecture compare to human perception

we observe the data from the learning dataset that match well with the data from the human perception. Some mistakes can be observed (like on texture $a$ ). We suppose they come from skids during the acquisition. To exclude this problem, the silicone surface needs to be change for a less sticky one. In blue, the test data are mostly different from one roughness grade compared to the human perception. Nevertheless, an exception is present: the texture $k$ roughness estimation from the algorithm has an error of 3 grades compared to what the humans perceived.

\section{CONCLUSION}

This study compared human roughness perception to a learning algorithm that was based on human roughness perception. Our first experiment with humans reveals the gap that can exist between different people's perception of roughness. In the second part of the study, we examined how our learning algorithm, using data from a tactile sensor and 
optimized by genetic algorithm, provided interesting results. Globally, they can be compared to the results obtained with humans. With new textures, the algorithm makes some small mistakes but keeps the right direction. There are two interesting innovative aspects in this study: first, it's one of the first studies that used humans as references to characterize a texture property. Secondly, we used genetic algorithm to optimize the learning algorithm properties. Many learning algorithms are used but often the parameters are set with default values or improve with different iterative tests. Using a genetic algorithm is an interesting option to optimize parameters to improve the results. Further research should focus on experiments with more textures to create an algorithm that is more reliable with all textures.

\section{ACKNOWLEDGEMENT}

The authors would like to acknowledge NSERC of Canada for funding and resources.

\section{REFERENCES}

[1] L. Natale, G. Metta, and G. Sandini, "Learning haptic representation of objects," in International Conference on Intelligent Manipulation and Grasping, 2004, Conference Proceedings.

[2] R. S. Dahiya, G. Metta, M. Valle, and G. Sandini, "Tactile sensing-from humans to humanoids," IEEE Transactions on Robotics, vol. 26, no. 1, pp. 1-20, 2010.

[3] M. R. Cutkosky, R. D. Howe, and W. R. Provancher, "Force and tactile sensors," Springer Handbook of Robotics, pp. 455-476, 2008.

[4] J. Engel, J. Chen, and C. Liu, "Development of polyimide flexible tactile sensor skin," Journal of Micromechanics and Microengineering, vol. 13 , no. 3 , p. 359,2003

[5] J. Ulmen and M. Cutkosky, "A robust, low-cost and low-noise artificial skin for human-friendly robots," in Robotics and Automation (ICRA), 2010 IEEE International Conference on. IEEE, 2010, pp. 4836-4841.

[6] A. Schmitz, M. Maggiali, L. Natale, B. Bonino, and G. Metta, "A tactile sensor for the fingertips of the humanoid robot icub," in Intelligent Robots and Systems (IROS), 2010 IEEE/RSJ International Conference on. IEEE, 2010, pp. 2212-2217.

[7] A. Rana, J.-P. Roberge, and V. Duchaine, "An improved soft dielectric for a highly sensitive capacitive tactile sensor," IEEE Sensors Journal, vol. 16, no. 22, pp. 7853-7863, 2016.

[8] L. Seminara, M. Capurro, P. Cirillo, G. Cannata, and M. Valle, "Electromechanical characterization of piezoelectric pvdf polymer films for tactile sensors in robotics applications," Sensors and Actuators A: Physical, vol. 169, no. 1, pp. 49-58, 2011.

[9] T.-H.-L. Le, A. Maslyczyk, and V. Duchaine, "A highly sensitive multimodal capacitive tactile sensor," Robotics and Automation (ICRA), 2017 IEEE International Conference on, pp. 999-999, 2017.

[10] J. A. Fishel and G. E. Loeb, "Sensing tactile microvibrations with the biotac-comparison with human sensitivity," in Biomedical Robotics and Biomechatronics (BioRob), 2012 4th IEEE RAS \& EMBS International Conference on. IEEE, 2012, pp. 1122-1127.

[11] V. Duchaine, "Why tactile intelligence is the future of robotic grasping," IEEE Spectrum, 2016.

[12] B. Heyneman and M. R. Cutkosky, "Slip interface classification through tactile signal coherence," in Intelligent Robots and Systems (IROS), 2013 IEEE/RSJ International Conference on. IEEE, 2013, pp. 801-808

[13] J.-P. Roberge, S. Rispal, T. Wong, and V. Duchaine, "Unsupervised feature learning for classifying dynamic tactile events using sparse coding," in Robotics and Automation (ICRA), 2016 IEEE International Conference on. IEEE, 2016, pp. 2675-2681.

[14] D. Cockburn, J.-P. Roberge, and V. Duchaine, "Grasp stability assessment through unsupervised feature learning of tactile images," Robotics and Automation (ICRA), 2017 IEEE International Conference on, pp. 999-999, 2017.

[15] W. Mayol-Cuevas, J. Juarez-Guerrero, and S. Munoz-Gutierrez, "A first approach to tactile texture recognition," in Systems, Man, and Cybernetics, 1998. 1998 IEEE International Conference on, vol. 5. IEEE, 1998, pp. 4246-4250.
[16] J. A. Fishel and G. E. Loeb, "Bayesian exploration for intelligen identification of textures," Frontiers in neurorobotics, vol. 6, 2012.

[17] D. Xu, G. E. Loeb, and J. A. Fishel, "Tactile identification of objects using bayesian exploration," in Robotics and Automation (ICRA), 2013 IEEE International Conference on. IEEE, 2013, pp. 3056-3061.

[18] N. Myshkin, A. Y. Grigoriev, S. Chizhik, K. Choi, and M. Petrokovets, "Surface roughness and texture analysis in microscale," Wear, vol. 254 , no. 10, pp. 1001-1009, 2003.

[19] T. Yoshioka, J. C. Craig, G. C. Beck, and S. S. Hsiao, "Perceptual constancy of texture roughness in the tactile system," Journal of Neuroscience, vol. 31, no. 48, pp. 17603-17611, 2011.

[20] W. McMahan and K. J. Kuchenbecker, "Dynamic modeling and control of voice-coil actuators for high-fidelity display of haptic vibrations," in Haptics Symposium (HAPTICS), 2014 IEEE. IEEE, 2014, pp. 115-122.

[21] A. B. Vallbo and R. Johansson, "Properties of cutaneous mechanoreceptors in the human hand related to touch sensation," Hum Neurobiol, vol. 3, no. 1, pp. 3-14, 1984.

[22] N. Myers, "Characterization of surface roughness," Wear, vol. 5, no. 3, pp. 182-189, 1962.

[23] A. Majumdar and B. Bhushan, "Role of fractal geometry in roughness characterization and contact mechanics of surfaces," Journal of Tribology, vol. 112, no. 2, pp. 205-216, 1990

[24] S. J. Lederman and R. L. Klatzky, "Haptic perception: A tutorial," Attention, Perception, \& Psychophysics, vol. 71, no. 7, pp. 1439-1459, 2009.

[25] C. J. Cascio and K. Sathian, "Temporal cues contribute to tactile perception of roughness," The Journal of Neuroscience, vol. 21, no. 14, pp. 5289-5296, 2001.

[26] R. S. Johansson and J. R. Flanagan, "Coding and use of tactile signals from the fingertips in object manipulation tasks," Nature Reviews Neuroscience, vol. 10, no. 5, pp. 345-359, 2009.

[27] G. Savelsbergh, B. Steenbergen, and J. Van der Kamp, "The role of fragility information in the guidance of the precision grip," Human Movement Science, vol. 15, no. 1, pp. 115-127, 1996.

[28] W. Mayol-Cuevas, J. Juarez-Guerrero, and S. Munoz-Gutierrez, "A first approach to tactile texture recognition," in Systems, Man, and Cybernetics, 1998. 1998 IEEE International Conference on, vol. 5. IEEE, 1998, Conference Proceedings, pp. 4246-4250.

[29] N. Jamali and C. Sammut, "Material classification by tactile sensing using surface textures," in Robotics and Automation (ICRA), 2010 IEEE International Conference on. IEEE, 2010, Conference Proceedings, pp. 2336-2341.

[30] _ _ "Majority voting: material classification by tactile sensing using surface texture," Robotics, IEEE Transactions on, vol. 27, no. 3, pp. 508-521, 2011.

[31] J. A. Fishel and G. E. Loeb, "Bayesian exploration for intelligent identification of textures," Frontiers in Neurorobotics, vol. 6, 2012.

[32] S.-H. Kim, J. Engel, C. Liu, and D. L. Jones, "Texture classification using a polymer-based mems tactile sensor," Journal of micromechanics and Microengineering, vol. 15, no. 5, p. 912, 2005.

[33] F. De Boissieu, C. Godin, B. Guilhamat, D. David, C. Serviere, and D. Baudois, "Tactile texture recognition with a 3-axial force mems integrated artificial finger," in Robotics: Science and Systems, 2009, Conference Proceedings

[34] A. M. Mazid and A. Ali, "Opto-tactile sensor for surface texture pattern identification using support vector machine," in Control, Automation, Robotics and Vision, 2008. ICARCV 2008. 10th International Conference on. IEEE, 2008, Conference Proceedings, pp. 1830-1835.

[35] D. S. Chathuranga, V. A. Ho, and S. Hirai, "Investigation of a biomimetic fingertip's ability to discriminate fabrics based on surface textures," in Advanced Intelligent Mechatronics (AIM), 2013 IEEE/ASME International Conference on. IEEE, 2013, Conference Proceedings, pp. 1667-1674.

[36] S. Takamuku, G. Gomez, K. Hosoda, and R. Pfeifer, "Haptic discrimination of material properties by a robotic hand," in Development and Learning, 2007. ICDL 2007. IEEE 6th International Conference on. IEEE, 2007, Conference Proceedings, pp. 1-6.

[37] A. M. Smith, C. E. Chapman, M. Deslandes, J.-S. Langlais, and M.P. Thibodeau, "Role of friction and tangential force variation in the subjective scaling of tactile roughness," Experimental Brain Research, vol. 144, no. 2, pp. 211-223, 2002. 